\title{
Cervical Meningeal Chondroma: A Case Report
}

Soichiro Takamiya' ${ }^{1}$, Toshitaka Seki', Kikutaro Tokairin' ${ }^{1}$, Syuji Hamauchi' ${ }^{1}$, Toru Sasamori', Tomoko Mitsuhashi², Kiyohiro Houkin ${ }^{1}$

${ }^{1}$ Department of Neurosurgery, Hokkaido University Graduate School of Medicine, N-15, W-7, Kita-ku, Sapporo, Hokkaido 060-8638, Japan

${ }^{2}$ Department of Surgical Pathology, Hokkaido University Hospital, N-14, W-5, Kita-ku, Sapporo, Hokkaido 060-8648, Japan

\begin{abstract}
Spinal chondromas are rare tumors, usually classified as periosteal chondroma or enchondroma, according to their origin. We describe a rare case of cervical spine meningeal chondroma in a 79-year-old man, who presented with right-sided weakness and lower-extremity dysesthesia. Magnetic resonance imaging revealed a tumor at the $\mathrm{C} 1-\mathrm{C} 2$ level. We suspected it to be meningioma and performed tumor excision. However, the final pathology diagnosed it as a meningeal chondroma. After surgery, his symptoms gradually improved. This case shows that, although uncommon, spinal chondromas may arise from the meninges. A good outcome is expected after total resection of the tumor.
\end{abstract}

Keywords: Cervical chondroma; Intradural extramedullary tumor; Meningioma; Meningeal chondroma; Spinal chondroma

\section{Introduction}

Spinal chondromas are rare, benign tumors. Most of them originate from the surface of the periosteum or the medullary cavity [1]; however, there are a few reports of spinal chondromas originating from the meninges [2,3]. Herein, we report a rare case of cervical spinal chondroma, which originated from the dura mater. Recognition that spinal chondromas may originate from the meninges is important because diagnosis can otherwise be difficult. The patient has given informed consent to publish this report.

\section{Case Report}

A 79-year-old male patient visited our outpatient clinic with progressive, right-side weakness and lower-extremity dysesthesia. His medical history was remarkable for dysesthesia of his upper extremities four years previously because of pyogenic spondylitis of C5-C6. Upon physical examination, there was a right side motor weakness (upper extremity: MMT4, lower extremity: MMT3). He also complained of dysesthesia in all extremities, which was especially pronounced on the right side. Cervical magnetic resonance imaging (MRI) revealed there was a spinal tumor with a cystic lesion on the right ventral side at the C1-C2 level (Figure 1A). The nodule showed a heterogeneously high signal intensity on T2-weighted imaging (Figure 1B) and T1-weighted imaging (Figure 1C), and a peripheral ring enhancement (Figure 1D). At first, we suspected it to be meningioma, schwannoma, solitary fibrous tumor, retro-odontoid pseudotumor, synovial cyst, or ganglion. C1-C2 laminectomy was performed, and the location of the tumor was confirmed by ultrasonography (Figure $2 \mathrm{~A}$ ). On opening the dura mater and the arachnoid mater, a white tumor could be seen on the ventral aspect of the dentate ligament (Figure 2B). The content of the cystic lesion of the tumor was a viscous, yellowish liquid (Figure 2C). Rapid intraoperative diagnosis of the cyst wall suggested it to be consistent with a meningioma. We then removed the brittle tumor, which seemed to be contiguous with the dura (Figure 2D), and cauterized the sections of the dura where the tumor originated. After surgery, the patient's symptoms slowly improved, especially the motor weakness of the right upper extremity and dysesthesia of the lower extremities. The final pathology report diagnosed the tumor as a meningeal chondroma. Hematoxylin and eosin staining revealed chondrocytes in the tumor (Figure 3A) with no obvious non-neoplastic meningeal tissue, and meningothelial cells in the tumor membrane (Figure $3 \mathrm{~B}$ ); these were not neoplastic but were histologically reactive. Immunostaining of the tumor for S-100 protein was positive (Figure 3C); CD68 was partially positive, and epithelial membrane antigen (EMA) was negative.

\section{Discussion}

Chondromas are a common benign tumor of cartilaginous tissue.
Their most common origins are long bones of the hands and feet, and spinal chondromas are quite rare, accounting for only $2 \%$ of all spinal tumors [4]. Most chondromas are classified as enchondromas (which arise from the medullary cavity), or periosteal chondromas (which arise from the periosteum) [1]. However, our case arose from the dura mater. There are some reports of intracranial chondroma originating from the dura mater, which have been called "meningeal chondroma" or "dural chondroma"; they account for $<0.3 \%$ of intracranial tumors [5]. However, there are even fewer reports of spinal chondroma originating from the dura mater [2,3]. To our knowledge, this is the third reported case of spinal meningeal chondroma.
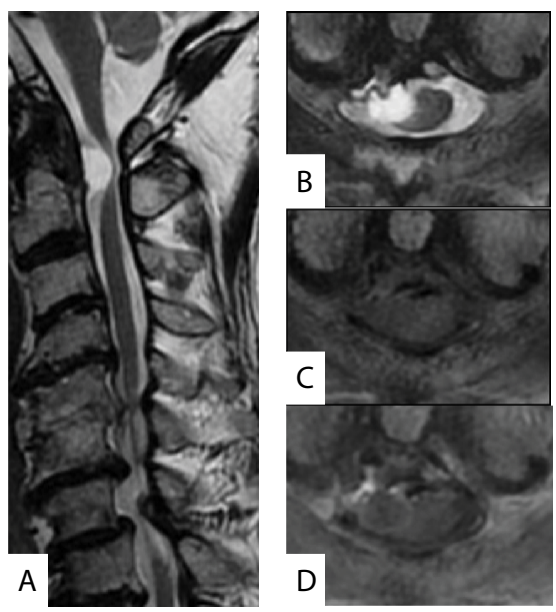

Figure 1: (A) T2-weighted sagittal magnetic resonance imaging (MRI) showed a spinal tumor with a cyst on the ventral aspect of the spine at the C1-C2 level. (B) The nodule, which existed ventral to the cystic lesion and seemed to be contiguous with the dura mater, was heterogeneously high signal intensity on T2-weighted axial MRI (C) T1-weighted imaging. (D) It had peripheral rim enhancement on gadolinium enhanced T1-weighted imaging.

${ }^{*}$ Corresponding author: Soichiro Takamiya, M.D., Department of Neurosurgery, Hokkaido University Graduate School of Medicine, N-15, W-7, Kita-ku, Sapporo, Hokkaido 060-8638, Japan, Tel: +81-11-706-5987; Fax: +81-11-708-7737; E-mail: soichiro.tkmy@gmail.com

Received September 24, 2016; Accepted October 13, 2016; Published October 15,2016

Citation: Takamiya S, Seki T, Tokairin K, Hamauchi S, Sasamori T, et al. (2016) Cervical Meningeal Chondroma: A Case Report. J Spine 5: 335. doi: 10.4172/2165 7939.1000335

Copyright: (c) 2016 Takamiya S. This is an open-access article distributed under the terms of the Creative Commons Attribution License, which permits unrestricted use, distribution, and reproduction in any medium, provided the original author and source are credited. 


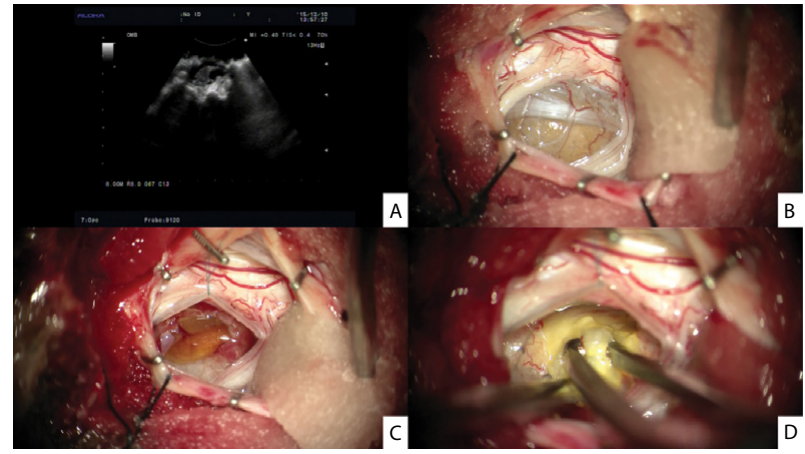

Figure 2: (A) Intraoperative findings of the spinal tumor excision. After C1C2 laminectomy, we performed ultrasonography to confirm accurately located the tumor. (B) When the dura mater and the arachnoid mater were opened, the white tumor was seen to be beyond the dentate ligament. (C) The cystic lesion of the tumor was composed of viscous, yellowish liquid, and (D) the tumor itself was brittle.

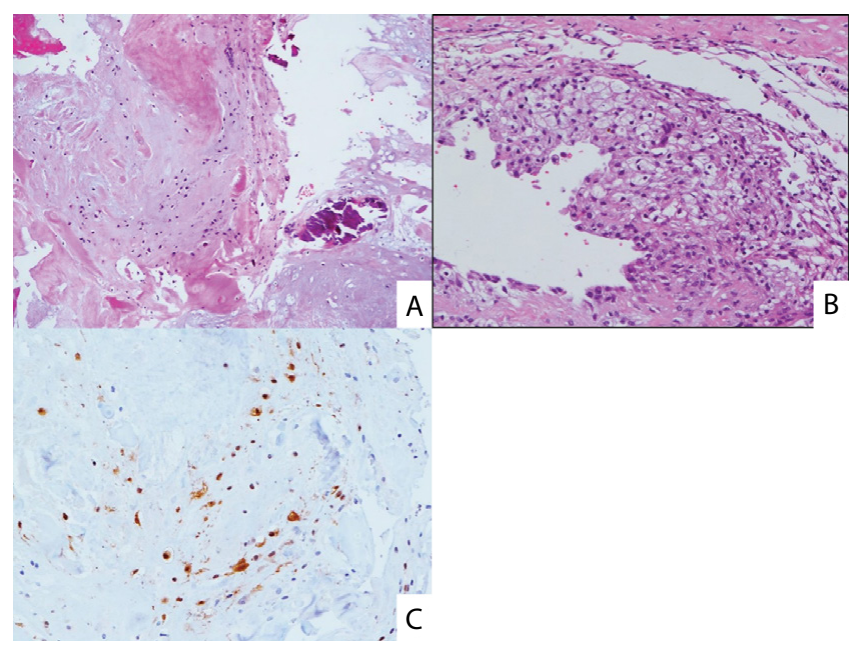

Figure 3: (A) Hematoxylin and eosin staining section of the excised tumor and (B) the membrane of the tumor. (C) Immunohistochemistry staining for S-100 protein was positive in tumor cells.
To our knowledge, only 16 reported cases of cervical chondroma (including our case) [1-3,6-17] (Table 1). Nine of the 16 cases (56\%) developed during the second and third decade of life, and rarely developed in senior citizens. Our patient was the oldest one among the 16 cases. Among the reported cases, 12 of 16 patients (75\%) were male. Moreover, there was a site predilection, the lower cervical spine (C4C7), fir the tumors in 11 of the 16 cases (69\%); thus, presentation in the upper cervical spine, as in our case, is rare. As described previously, the majority of tumors ( 13 cases, $81 \%$ ) were periosteal chondroma or enchondroma, and only three cases (19\%) were meningeal chondroma. Of interest, the characteristics of our case were very similar to that of the case reported by Byun et al. [2]. Both cases developed in older men, were located in the upper cervical spine, and were categorized as meningeal chondroma. There might be some common points in meningeal chondroma; however, the case reported by Raheje et al. [3] was entirely different.

Although it is difficult to diagnose meningeal chondroma on radiological findings alone, MRI give us some helpful information. They usually show heterogeneously high signal intensity on T2-weighted images and peripheral rim enhancement on gadolinium-enhanced T1-weighted images [3]. These findings are important to distinguish them from other tumors, in that there is no dural tail or homogeneous enhancement with gadolinium.

Histologically, spinal chondromas are composed of welldifferentiated chondrocytes in hyaline and myxoid matrix. They sometimes have varying degrees of calcification. Immunohistochemistry staining shows positive for S-100 protein and negative for epithelial markers such as EMA. In our case, meningothelial cells were presented in the membrane of the tumor, and we suspected it to be a meningioma. However, there was no evidence of neoplastic proliferation of the meningothelial cells, which were reactive. We also considered the possibility that a periosteal chondroma had invaded the subdural space through the dura mater, but there was no continuity between the tumor and cervical vertebrae on MRI. For these reasons, we diagnosed the tumor as a meningeal chondroma.

Total tumor excision is recommended for spinal chondromas. Chemotherapy is ineffective, but radiation therapy may be chosen if surgical resection is not possible [2]. Following complete resection of

\begin{tabular}{|c|c|c|c|c|c|c|c|}
\hline Author & Year & Age & Gender & Location & Category & Treatment & Recurrence \\
\hline Present case & 2015 & 79 & $M$ & $\mathrm{C} 1$ & Meningeal Chondroma & Complete tum or resection & No \\
\hline Byan et al. [2] & 2015 & 72 & M & C1-C2 & Meningeal Chondroma & Complete tum or resection & No \\
\hline Raheja et al. [3] & 2015 & 25 & M & C4-C5 & Meningeal Chondroma & Partial tum or resection & No \\
\hline Jeong et al. [7] & 2014 & 24 & $\mathrm{~F}$ & C4-C5 & Enchondroma & Complete tum or resection & No \\
\hline Wani et al. [8] & 2011 & 25 & M & $\mathrm{C} 7$ & Periosteal Chondroma & Complete tum or resection & No \\
\hline Russo et al. [9] & 2010 & 38 & M & C5 & Periosteal Chondroma & Complete tum or resection & No \\
\hline Fahim et al. [1] & 2009 & 6 & M & C5-C6 & Periosteal Chondroma & Complete tum or resection & No \\
\hline Shurland et al. [10] & 1999 & 11 & $\mathrm{~F}$ & C5 & Enchondroma & Tum or curettage & No \\
\hline Antic et al. [11] & 1992 & 28 & M & C5-C6 & Periosteal Chondroma & Complete tum or resection & No \\
\hline Baber et a1. [12] & 1988 & 50 & M & C4-C5 & Periosteal Chondroma & En-block excision & No \\
\hline palaoglu et al.[13] & 1988 & 30 & M & C5-C6 & Periosteal Chondroma & Tum or curettage & Yes \\
\hline Loges et al. [6] & 1987 & 76 & $\mathrm{~F}$ & C4-C5 & Periosteal Chondroma & Partial tum or resection & No \\
\hline Willis et al. [14] & 1986 & 24 & M & C3-C5 & Enchondroma & En block excision & No \\
\hline Calderone et al. [15] & 1982 & 20 & M & $\mathrm{C} 2$ & Periosteal Chondroma & Tum or curettage & Not described \\
\hline Maiuri et al. [16] & 1980 & 20 & M & $\mathrm{C} 2-\mathrm{C} 3$ & Periosteal Chondroma & Partial tum or resection & No \\
\hline slowik et al. [17] & 1968 & 10 & $\mathrm{~F}$ & $\mathrm{C} 6-\mathrm{C} 7$ & Periosteal Chondroma & Complete tum or resection & No \\
\hline
\end{tabular}

Table 1: 16 published cases of cervical spine chondroma. 
the tumor, the recurrence rate is $<10 \%$ [18]. As seen in the 16 cases of cervical chondroma (Table 1), the recurrence rate was $0 \%$ when the tumor could be resected completely, although it was $6.3 \%$ when the tumor could not be resected completely.

\section{Conclusion}

We reported a rare case of spinal meningeal chondroma. These tumors are difficult to diagnose because they usually do not originate from meninges. Total excision of the tumor is required, and a complete recovery is expected.

\section{Conflict of Interest Disclosure}

No potential conflict of interest relevant to this article was reported.

\section{References}

1. Fahim DK, Johnson KK, Whitehead WE, Curry DJ, Luerssen TG, et al. (2009) Periosteal chondroma of the pediatric cervical spine. J Neurosurg Pediatr 3 : 151-156.

2. Byun YH, Sohn S, Park SH, Chung CK (2015) Cervical spine chondroma compressing spinal cord: A case report and literature review. Korean J Spine 12: $275-278$

3. Raheja A, Borkar SA, Nalwa A, Suri V (2015) Primary spinal extraosseous cervical chondroma in an adult. Neurol India 63: 114-116.

4. Thien A, Teo CH, Lim CC, Karandikar A, Dinesh SK (2014) Soft tissue chondroma mimicking "dumbbell" neurogenic tumor: a rare cause of lumbar radiculopathy. J Clin Neurosci 21: 1073-1074.

5. Kumari N, Sahu RN, Krishnani N (2010) Meningeal chondroma in a young female. Indian J Pathol Microbiol 53: 117-118.

6. Jeong DM, Paeng SH (2015) Enchondroma of the cervical spine in young woman: A rare case report. Asian J Neurosurg 10: 334-337.
7. Wani AA, Zargar JI, Ramzan AU, Malik NK, Lone I, et al. (2011) Isolated enchondroma of atlas. Turk Neurosurg 21: 226-229.

8. Russo V, Platania N, Graziano F, Albanese V (2010) Cervical spine chondroma arising from $\mathrm{C} 5$ right hemilamina: a rare cause of spinal cord compression. Case report and review of the literature. J Neurosurg Sci 54: 113-117.

9. Shurland AT, Flynn JM, Heller GD, Golden JA (1999) Tumor of the cervical spine in an 11-year-old. Clin Orthop Relat Res 368: 287-290, 293-295.

10. Antic B, Roganovic Z, Tadic R, llic S (1992) Chondroma of the cervical spina canal. Case report. J Neurosurg Sci 36: 239-241.

11. Baber WW, Numaguchi Y, Kenning JA, Harkin JC (1988) Periosteal chondroma of the cervical spine: one more cause of neural foramen enlargement. Surg Neurol 29: 149-152.

12. Palaoglu S, Akkas O, Sav A (1988) Chondroma of the cervical spine. Clin Neurol Neurosurg 90: 253-255.

13. Lozes G, Fawaz A, Perper H, Jomin M (1987) Chondroma of the cervical spine. Case report. J Neurosurg 66: 128-130.

14. Willis BK, Heilbrun MP (1986) Enchondroma of the cervical spine. Neurosurgery 19: $437-440$

15. Calderone A, Naimark A, Schiller AL (1982) Case report 196: juxtacortical chondroma of C2. Skeletal Radiol 2: 160-163.

16. Maiuri F, Corriero G, De Chiara A, Giamundo A, Benvenuti D, et al. (1980) Chondroma of the cervical spine: a case report. Acta Neurol (Napoli) 2: 204-208.

17. Slowik T, Bittner-Manioka M, Grochowski W (1968) Case reports and technica notes. Chondroma of the cervical spine. Case report. J Neurosurg 29: 276-279.

18. Morard M, De Tribolet N, Janzer RC (1993) Chondromas of the spine: report of two cases and review of the literature. $\mathrm{Br} \mathrm{J}$ Neurosurg 7: 551-556. 